\title{
Dual Protection Use to Prevent STIs and Unintended Pregnancy
}

\author{
Jessica M. Sales, ${ }^{1}$ Maura K. Whiteman, ${ }^{2}$ Melissa J. Kottke, ${ }^{3}$ Tessa Madden, ${ }^{4}$ and Ralph J. \\ DiClemente $^{1}$
}

${ }^{1}$ Department of Behavioral Sciences and Health Education, Rollins School of Public Health, Emory University, 1518 Clifton Road NE, Atlanta, GA 30322, USA

${ }^{2}$ Division of Reproductive Health, National Center for Chronic Disease Prevention and Health Promotion, Centers for Disease Control and Prevention, Atlenta, GA 30341, USA

${ }^{3}$ School of Medicine, Emory University, Atlenta, GA 30322, USA

${ }^{4}$ Washington University School of Medicine, St. Louis, MO 63110, USA

Correspondence should be addressed to Jessica M. Sales, jmcderm@emory.edu

Received 24 December 2011; Accepted 24 December 2011

Copyright ( $) 2012$ Jessica M. Sales et al. This is an open access article distributed under the Creative Commons Attribution License, which permits unrestricted use, distribution, and reproduction in any medium, provided the original work is properly cited.

Young women are at greatest risk for unintended pregnancy and contracting a variety of sexually transmitted infections (STIs). For over a decade, medical practitioners, researchers, and professional organizations have recommended comprehensive reproductive health counseling for young women which includes recommending dual protection strategies designed to enhance prevention of both unintended pregnancy and STI/HIV. Although recommended, dual protection is not commonly practiced, especially in the United States. Thus, this special issue presents research articles and review articles to identify and better understand factors influencing dual protection use.

This special issue contains eight papers. Three papers present findings on dual protection use among adolescents and adults in the United States, and two papers report findings on dual protection use among HIV-positive individuals in international settings (Zambia and India). In addition to research articles, three review papers report on biomedical approaches to dual protection, the potential impact of multiple risk messaging on condom use among women, and biological strategies to enhance the measurement of dual protection.

In the article titled "Correlates of dual-method contraceptive use: an analysis of the national survey of family growth (2006-2008)", D. L. Eisenberg et al. report on correlates of dual contraceptive use among a nationally representative sample of women aged 15-44 living in the United States. Dual contraceptive use was only reported by $7.3 \%$ of the 5,178 sexually active women in the sample. Correlates of dual contraceptive use included younger age, nonmarried marital status, and continuous health insurance in the past 12 months.

J. L. Brown et al., in their article titled "Multiple method contraception use among African American adolescents in four US cities," describe the contraceptive practices and type of contraception utilized during the last sexual episode reported by African American adolescents in the United States. They found that $40 \%$ used dual (or multiple) contraceptive methods, with a total of 35 different contraceptive combinations identified. Adolescents who believed their partners posed low risk with regard to STI acquisition were more likely to use less effective contraceptive methods (e.g., withdrawal) or to use no contraception.

In the article titled "Determinants of multimethod contraceptive use in a sample of adolescent women diagnosed with psychological disorders," D. L. Lang et al. examined individual-, interpersonal-, and environmental-level factors associated with multimethod use among sexually active adolescent women diagnosed with psychological disorders. Among this subgroup of adolescents, $34.7 \%$ of the participants reported 
multimethod use in the past three months. In controlled analyses, self-efficacy, multiple partners, pregnancy history, parental communication, parental norms about sex, and neighborhood cohesion were significant predictors of multimethod use.

C. J. Chibwesha et al. present findings pertaining to dual contraceptive use among HIV-infected women in their article, "Modern contraceptive and dual method use among HIVinfected women in Lusaka, Zambia." In this article the authors describe the implementation of a reproductive health counseling intervention conducted in 16 HIV clinics in Lusaka, Zambia. Of the 18,407 women counseled, 59\% reported current modern contraceptive use but only $18 \%$ reported dual contraceptive use. After counseling, many women not previously using modern contraception desired family planning referrals and successfully accessed services within 90 days.

In the research article titled "Prevalence of and barriers to dual-contraceptive methods use among married men and women living with HIV in India," V. Chakrapani et al. describe the prevalence and correlates of dual-contraceptive methods use and barriers to their use among married persons living with HIV in India. Utilizing a mixed-method approa$\mathrm{ch}$, they found the prevalence of dual-contraceptive method use increased from 5\% before HIV diagnosis to $23 \%$ after diagnosis. Condoms were the most commonly used contraceptive method, with prevalence increasing from 13\% before diagnosis to $92 \%$ after diagnosis. Barriers to using contraceptive methods other than condoms included lack of discussion about other methods by health care providers, lack of acceptability of other methods, and lack of involvement of husbands in family planning counseling.

In the review paper titled "Multipurpose prevention technologies: biomedical tools to prevent HIV-1, HSV-2, and unintended pregnancies," A. R. Thurman et al. provide an overview of existing and novel approaches to multipurpose products that provide simultaneous protection against both unintended pregnancies and STIs. For instance, they discuss how potent antiviral drugs released topically in the female reproductive tract by innovative delivered systems and formulations may provide safe, effective, and acceptable multipurpose prevention tools.

In the article titled "Are dual-method messages undermining STI/HIV prevention?” A. O'Leary provides an overview of the literature on multiple risk messages, compliance with dual-use recommendations, predictors of dual use, and interventions developed to encourage dual use. She argues that because simultaneous use of dual methods is not common, and efforts to enhance dual use have not yielded promising results, a randomized controlled trial to explore the impact of multiple risk messaging on condom use among young women should be considered.

In the review titled "Do women using long-acting reversible contraception reduce condom use? A novel study design incorporating semen biomarkers," M. F. Gallo et al. review evidence from previous studies of condom "migration" associated with the use of long-acting reversible contraceptive methods and propose a novel study design to address the main methodological issues that have limited interpretation of these studies. Specifically, they propose a randomized controlled trial design utilizing the use a biological marker of semen exposure for measuring changes in condom use to overcome methodological issues.

\section{Disclosure}

The findings and conclusions in this report are those of the authors and do not necessarily represent the official position of the Centers for Disease Control and Prevention.

Jessica M. Sales

Maura K. Whiteman

Melissa J. Kottke

Tessa Madden

Ralph J. DiClemente 


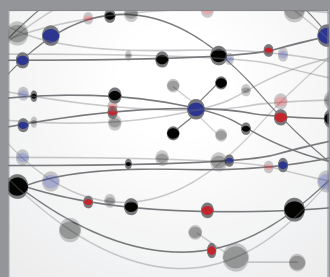

The Scientific World Journal
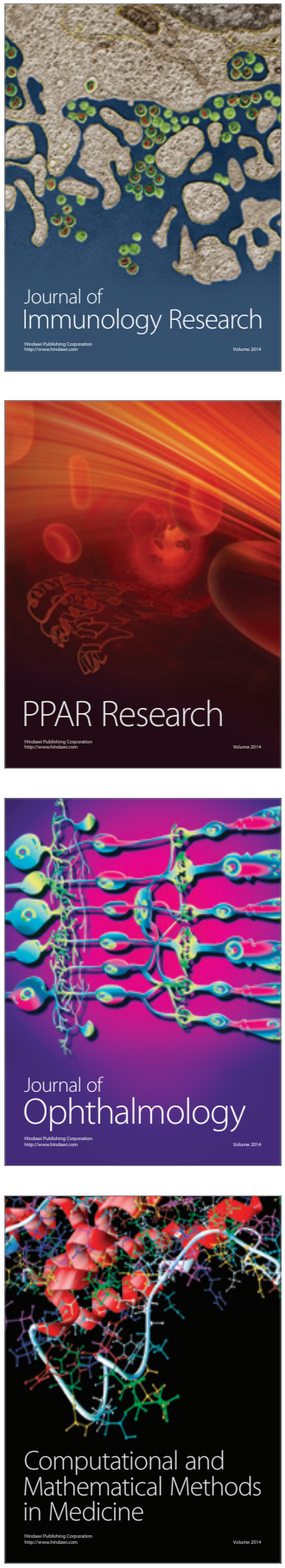

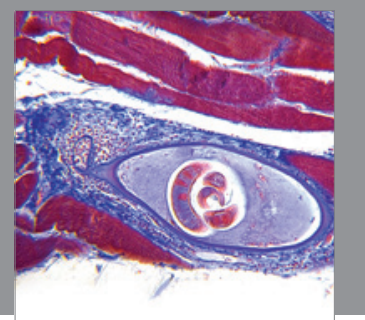

Gastroenterology

Research and Practice
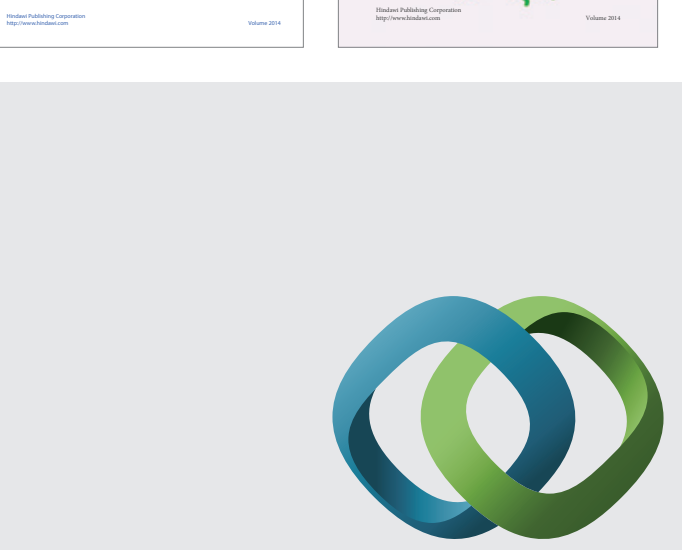

\section{Hindawi}

Submit your manuscripts at

http://www.hindawi.com
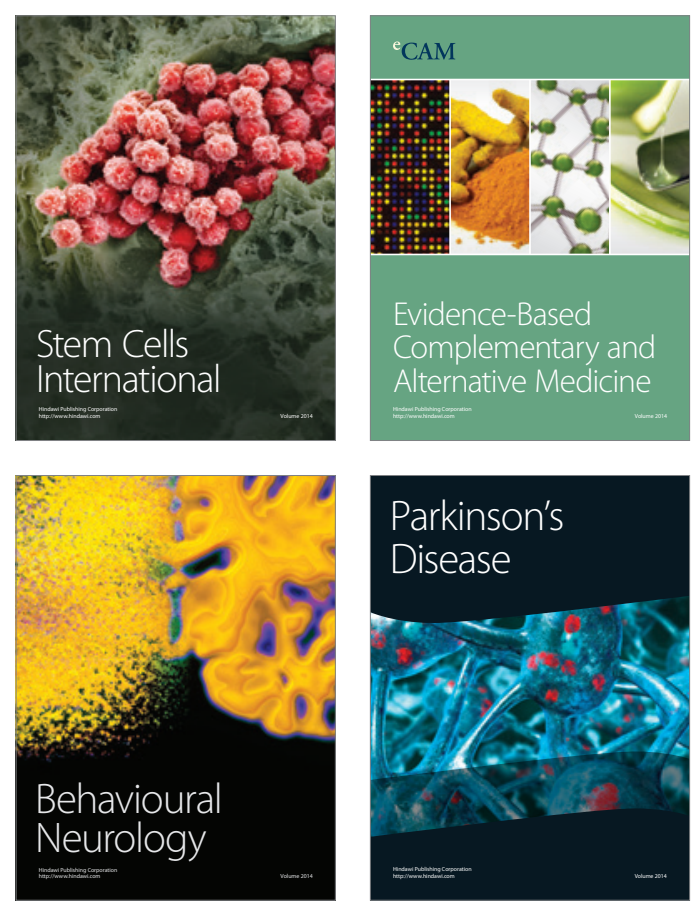

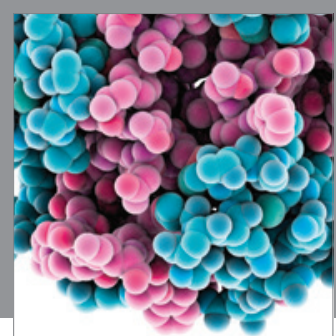

Journal of
Diabetes Research

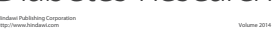

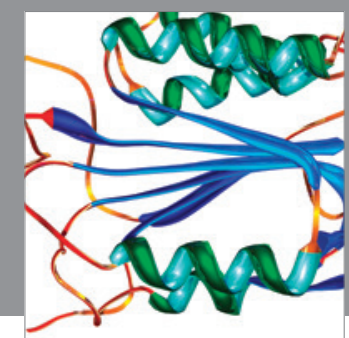

Disease Markers
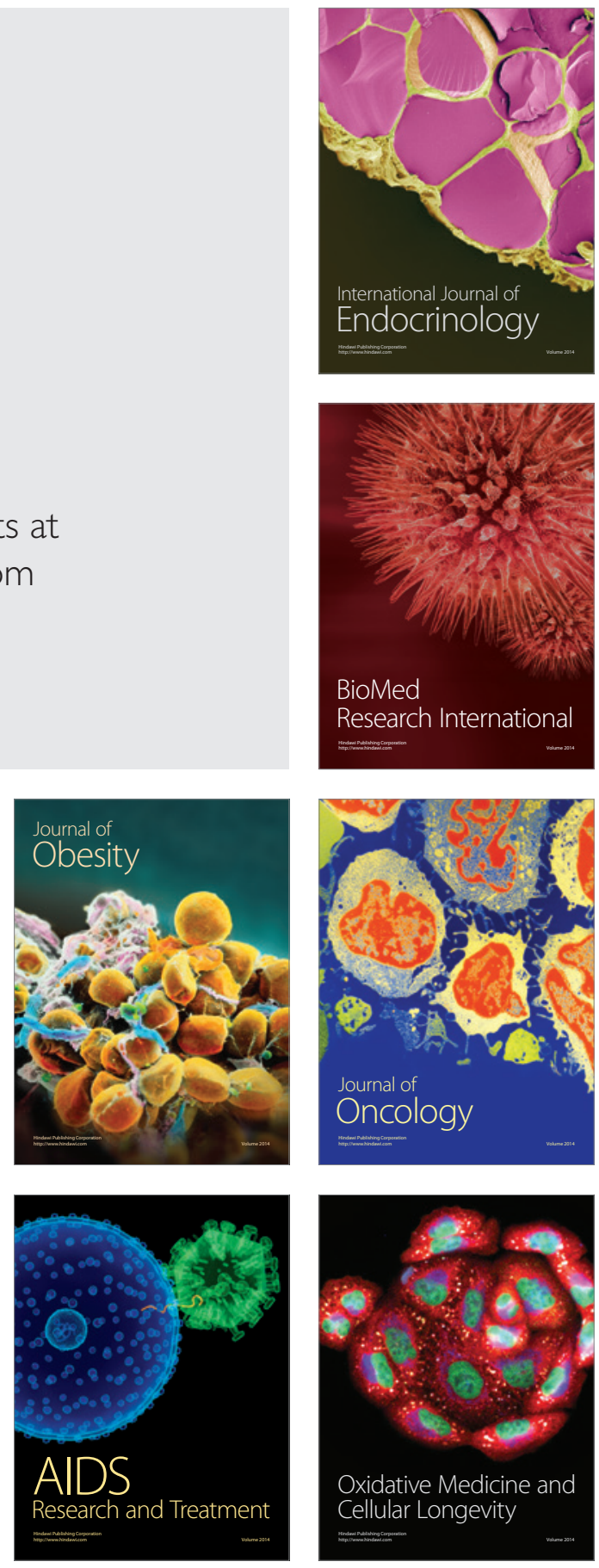